\title{
Second Life in Healthcare Education: Virtual Environment's Potential to Improve Patient Safety
}

\author{
Angie Lee \\ Department of Education \\ University of Maryland Baltimore County (UMBC) \\ 1000 Hilltop Circle, Baltimore, MD, 21045, USA
}

\author{
Zane L. Berge* \\ Department of Education \\ University of Maryland Baltimore County (UMBC) \\ 1000 Hilltop Circle, Baltimore, MD, 21045, USA \\ E-mail: berge@umbc.edu \\ *Corresponding author
}

\begin{abstract}
Research has shown that a large portion of healthcare cost is due to medical errors. There are many factors that cause medical errors but one major factor is linked to healthcare professionals who are not adequately trained with the appropriate skills. Virtual environments and simulations are being used to help improve training in many different areas, including the healthcare profession. This paper explores the usage of Second Life in healthcare education and its ability to improve patient safety. The conclusion is that much progress and development still needs to be made before Second Life will make a significant impact on improved patient safety through healthcare education. There is a need to make the virtual environment more realistic to adequately train healthcare professionals.
\end{abstract}

Keywords: Second Life; Virtual environments; Patient safety

Biographical notes: Zane L. Berge is Professor and former Director of the Training Systems, Instructional Systems Development Graduate Program at the University of Maryland Baltimore County. Between January 1992 and July 1995, Dr. Berge served as Director of the Center for Teaching and Technology and Assistant Director for Training Services, Academic Computer Center, Georgetown University. The mission of the Center is to promote the use of instructional technology in the classroom. Prior to Georgetown University, he was recruited in 1983 to Michigan State University in a highly competitive national competition for a fellowship program with the Institute for Research on Teaching. Prior to MSU, he held a variety of positions, ranging from Warehouse Group Leader to Vice President, in both large and small business organizations. This background has allowed accomplishments in the areas of instructional technology, instructional design, distance education, computers in training/education, marketing, sales, accounting, and general management. Education includes a BS (1977), with highest honors in Photo management/Business Administration from Rochester Institute of Technology; attended the University of Michigan Graduate School of Business in the MBA program, earned a Ph.D. in Educational Systems Development from Michigan State University (1988). majoring in computers in training and education; with minors in business; learning and cognition; and statistical methods. His 
research interest is distance education; mobile learning; and training in the workplace.

\section{Introduction}

The rising cost of healthcare is a major concern for many today. The increasing cost of healthcare has caused many to explore whether high medical costs are preventable or are they simply part of the nature of keeping people healthy. The Institute of Medicine estimated the cost of preventable adverse events in the United States to be \$17-29 billion per year (Mello, Studdert, Thomas, Yoon, \& Brennan, 2007). Billions of dollars are spent on healthcare due to medical errors that could be prevented. Besides the costliness of rectifying medical errors, there is also the immeasurable cost of a person's livelihood due to medical errors. There are many reasons for medical errors and it is often difficult to identify one source. However, according to Kohn et al. (2000), significant factors for a safe healthcare environment include having "the right equipment, well-maintained and reliable; a skilled and knowledgeable workforce; reasonable work schedules, welldesigned jobs; clear guidance on desired and undesired performance, et cetera.".

Skill and knowledge begins with healthcare education and training. How well trained and adequate are the skills of healthcare professionals affects patient safety. This paper explores the usage of virtual environments to educate healthcare professionals and determine its effectiveness and viability for usage in healthcare education. The virtual environment focused on here is Second Life.

\section{Second Life}

Second Life (SL) is a 3-D, virtual environment created by Linden Labs (Gollub, 2010). Users of SL create avatars and are able to interact with other users through a virtual environment. This virtual environment is often used as a social networking platform, however, in recent years, many colleges and universities have used SL as an educational platform to engage students and to build virtual environments and scenarios that simulate the real world. The graphics in SL make the environment look rather game-like but for the most part, behind each avatar is a live person who is controlling the avatar's communication and actions. Communication can be through text or voice and is in realtime. As Wong (2006) describes avatars in SL, "there is a real human being behind every avatar -- the people are very real. It's just the medium is different.".

One of the advantages of Second Life is that users are able to build objects and program scripts within the virtual world. The objects one creates can look like a car, a house, medical devices, and basically anything you can imagine. The programmed scripts make the objects one creates perform different actions (Heaton, 2007). Advanced users of SL use scripts to make the object behave a certain way. For example, a user can create an object that looks like anything he or she wants, and write a script so that it looks like the user's avatar is driving the car. This is useful because it makes the objects within the virtual world fully customizable by the user.

Colleges and universities have built replicas of their schools within SL. They use the virtual environment for collaboration, discussions, office hours, lectures, and even create virtual experiences for their students (Waters, 2009). Schools of medicine, pharmacy and nursing are also using SL as part of their curriculum to help train their students. 


\section{Current Usage of Second Life in Healthcare Education}

One example of SL being used in healthcare education is at Imperial College London. They have built a hospital in SL where students can perform such tasks as seeing patients, ordering X-rays, consulting with colleagues and making diagnoses (Bradley, 2009). Students can access the hospital 24 hours a day and practice diagnosing pre-programmed patients. Modeling the routine of a normal hospital, students, as their avatars, are also guided through the steps necessary to begin seeing patients. The virtual hospital is very large and even consists of an operating room area and recovery area. Within the operating room area, there are directions along the pathway that guide one through the proper steps of entering into a surgery room such as getting into uniform, wearing a mask and scrubbing down. Once one has performed those functions, he or she is allowed to enter into an operating room with operating room equipment and descriptors for each part of the operating room describing its functionality (Hastings, 2008).

In the recovery area at Imperial College London, they have created a chatbot that will respond to comments through text. A person's avatar in SL types questions or comments to the chatbot and the chatbot will respond. This type of development of virtual patients that can converse with students in a natural conversation has been an important part of deepening the immersive experience leading to better achieve the learning outcomes (Danforth, Procter, Heller, Chen, \& Johnson, 2009). Many of these chatbots use open source programming language that uses pattern matching and templates to interpret the input and to execute an output. Imperial College London is currently using a chatbot to elicit feedback from visitors. Currently, this chatbot cannot functionally be used to practice clinical skills.

A second example of SL being used in healthcare education is from the University of North Carolina's (UNC) School of Pharmacy. UNC's Information Technology Services have replicated parts of UNC's pharmacy school in SL. They have also built replicas of hospitals and practice sites in which their students will be visiting during their experiential rotations. Students, in their SL avatars, can visit these places in SL to familiarize themselves with the environment before actually going in real life. For example, in SL, students can visit the virtual representation of Greensboro's Moses Cone Family Practice Center (Anonymous, 2009). Students are able to role-play in real time. One student is the pharmacist while the other student is given the case study of a patient and answers the pharmacist's questions in accordance with the predetermined condition of the patient. The student that is playing the pharmacist can then take a quiz inside the virtual environment based on the encounter to assess the interaction. The benefit of UNC's SL environment is that it allows students to become comfortable with the places that they will be going to in real life.

UNC School of Pharmacy is also working on creating virtual representations of medical devices. Some drug dispensing devices are expensive to purchase just for the pharmacy school and for pharmacy education. However, students are not able to receive training on different types of medical devices until they are actually at a site that uses that machine. In some cases, students will not have exposure to different types of machines until they have graduated and they are working in a pharmacy environment. Without being able to practice using these machines, students are not adequately trained which can lead to the medical errors we addressed earlier. Building virtual replicas of these machines and simulating how they operate will help students receive training and exposure before having to use the machine in a real-life situation. 
There is much that has been built in SL related to healthcare education. However, determining the effectiveness of the training is highly dependent on the users' experience. When students navigate through SL, is what they experience adequate enough training for them to apply in the real world? I visited the virtual places described to gather a user's perspective and to experience what a medical student or pharmacy student might experience at Imperial College London and UNC pharmacy school.

\section{User Experience in Second Life Healthcare Environments Imperial College London's Virtual Hospital}

I toured Imperial College London's virtual hospital to experience what it is like to be a student going through the process of accessing the different patient scenarios in the virtual hospital (Taylor, n.d.b). The following actions took place in the Imperial College London SL virtual hospital (Taylor, n.d.a). As I entered the virtual hospital, I was given a card with directions describing how to proceed through the area and a podcast began to play in the background explaining the history of why the virtual hospital was developed. I entered the first area and was instructed on the different steps I needed to follow in order to successfully complete my task in the virtual hospital. Points are given for each action that I did correctly. As I walked in, I first needed to change into my uniform. The avatar does not actually change clothes, but when I touched the lockers in the room, a script recognized that I had completed that task and I was allowed to continue. I was then required to relieve the nurse on duty and received instructions regarding which patient I was to treat. The virtual nurse was activated when I mouse clicked on her. I was told that there is a patient in bed who has been on an IV but has not been evaluated in awhile. I proceeded to tend to the patient by first washing my hands and checking the patient's chart. You then click on the patient to find out if there are any issues with the patient. The patient was scripted to tell me that she was not feeling well. I could then check the drugdispensing machine. If I did so, I was prompted to answer some questions such as what type of drug is the machine dispensing, and to then make a preliminary diagnostic of the patient. The patient then turn on her side, and the machine started to beep. To stop the machine's beeping sound; I had to click on the machine. The patient then told me she was feeling dizzy and ill. I was prompted to answer what I wanted to do, with the options of checking the patient's blood pressure and other activities. The correct response is to turn the patient on her back to stop the occlusion caused by the patient turning on her side, this pinching the tubes. At that point, I received 9/16 points and I could not figure out what to do to help the patient. This could be due to my lack of medical training; but, it also did not seem clear from the scenario and the patient was not programmed for me to be able to ask more questions. Overall, the experience was interesting, albeit not that realistic. Without any prior medical training, I learned some things about the importance of patient positioning and drug-dispensing machines. Conceptually, I was able to understand the basic concepts I needed to learn, but I did not learn the practical components such as how to actually operate the machine. Also, because of how the patient was programmed, I was not able to really have a conversation with the patient to be able to understand the full picture of what was going on.

\section{Imperial College London's Polyclinic}

I also visited Imperial College London's Polyclinic and had a conversation with their chatbot, BHealthy (Taylor, n.d.a). At times, the conversation would seem realistic and the chatbot would be able to identify key words and responds in a way that was human-like. 
However, unlike a real person, where one could carry a conversation, the chatbot would respond sentence by sentence. Also, many responses from the chatbot did not make sense given what I was saying. The chatbot would also have a lot of preprogrammed response such as its favorite color and when I told the chatbot that it is a robot, it agreed with me, saying it has a heart of silicon. I believe that further development needs to be made on the virtual patient to make the simulation more realistic and to make possible realistic, clinical skills practice. In any healthcare profession, communication with the patient is one of the key components in proper diagnosis. Proper diagnosis generally leads to proper patient care. If virtual patients are to be used to help train healthcare professionals to increase patient safety, the virtual patients will need to be programmed with more complex algorithms to be able to respond more human-like.

\section{UNC's Virtual Pharmacy}

I visited UNC's virtual pharmacy (UNC V, n.d.). The virtual pharmacy school had many buildings. Some of these buildings replicated clinical environments that UNC's pharmacy students would be encountering and some of the buildings were virtual replicas of UNC's pharmacy school (Anonymous, 2009). I visited the replica of Greensboro's Moses Cone Family Practice Center. As I entered, there was a waiting room; touching one of the buttons triggered an audio response giving directions concerning how to proceed through the center. The first room is a room with an introductory video on how to conduct a patient interview. In the second room, there are different case studies for the student who is supposed to act as a patient to read through and know how to respond. The room is designed so that one student role-plays a pharmacist, and can interview a student who is role-playing the patient. I then moved to an area where the student-pharmacist can answer questions to assess their previous interaction. This situation is more realistic than the hospital simulation mentioned above, since there is a real person behind the avatar playing the patient. That is to say, in this simulation, the conversation between two real people, and therefore questions can be answered in a realistic way. The avatars lacked facial expressions and body language however, so the expressiveness could only be at a rather low level during the interview.

\section{Drug-Dispensing Machine}

I also visited the large virtual model of a drug-dispensing machine created by UNC pharmacy school in SL. The machine had buttons that your avatar could push and a dialog box would appear describing to you what each button does. Some of the controls were not push buttons but dials and levers. Unfortunately, most avatars are not scripted to do those types of motions to control the machine. During an interview with the creator of the virtual environment, she shared that most students will not encounter this machine until they are also participating in their experiential rotations. The idea is that students having a virtual exposure to the instrument will help to prepare them for their real life experience with the device. The virtual device was still in development mode, so with more scripting and editing the device can be more realistic and better suited for training.

\section{Conclusions}

Second Life is currently being actively used in healthcare education as demonstrated by the examples described above. However, there is still much development that needs to be done before it will make a major impact on healthcare training and improving patient safety. Some skills that can be improved through the usage of virtual environments are 
critical thinking and problem solving skills. For the foreseeable future, communication skills will probably be learned best through face to face interaction. Healthcare professional and patient interactions can be complex and is very challenging to write a script to simulate that type of dynamic relationship. Also, in order for healthcare professionals to receive adequate training, they need to be familiar with how to operate certain equipment. This type of training is hands-on. Still, a good deal of preparing for real life experience with instruments can be done virtually beforehand using simulations in such virtual worlds as Second Life.

Second Life in healthcare education is very valuable as a supplemental tool to the traditional healthcare training and probably will become more so in the future as simulations become better. For virtual environments to continue to grow and make a positive impact on healthcare education, virtual environments need to be more realistic for students. As medical errors and patient safety is a matter of life or death, simulation needs to mimic real life more closely. Without this type of development, Second Life will continue to be suitable as only a supplemental tool rather than a viable, life-changing educational tool that contributes to the healthcare profession.

\section{References}

1. Anonymous. (2009). Second Life may put pharmacy students on fast track. UNC Information technology services. Retrieved from http://its.unc.edu/InsideITS/ insideitsnews/CCM1 010995.

2. Bradley, J. (2009). Can Second Life help doctors to treat patients? In CNN. Retrieved from http://www.cnn.com/2009/TECH/03/30/doctors.second.life/.

3. Danforth, D., Procter, M., Heller, R., Chen, R., \& Johnson, M. (2009). Development of virtual patient simulations for medical education. Journal of virtual worlds research, 2(2), 1-11.

4. Gollub, R. (2010). Second life and education. Retrieved from http://www.acm.org/crossroads/xrds14-1/secondlife.html.

5. Hastings, H. (2008). Exploring medical training in Second Life - Imperial College London's virtual medical school. In CNN iReport. Retrieved from http://www.ireport.com/docs/DOC-159220.

6. Heaton, J. (2007). Programming Second Life with the Linden scripting language. Retrieved from http://www.devx.com/opensource/Article/33905.

7. Kohn, L.T., Corrigan, J.M., \& Donaldson, M.S. (2000). To err is human: Building a safer health system. Retrieved from http://www.nap.edu/ catalog.php?record_id=9728\#toc.

8. Mello, M.M., Studdert, D.M., Thomas, E.J., Yoon, C.S., \& Brennan, T.A. (2007). Who pays for medical errors? An analysis of adverse event costs, the medical liability system, and incentives for patient safety improvement. Journal of Empirical Legal Studies, 4(4), 835-860.

9. Taylor, D. (n.d.a). Health Lands. In Imperial College London. Retrieved from http://slurl.com/secondlife/HealthLands/165/158/32.

10. Taylor, D. (n.d.b). Second Health London. In Imperial College London. Retrieved from http://slurl.com/secondlife/Imperial\%20College\%20London/150/86/27/. 
11. UNC V. (n.d.). University of North Carolina Chapel Hill. Retrieved from http://slurl.com/secondlife/UNCV/174/92/28.

12. Waters, J.K. (2009). A 'Second Life' for educators. THE Journal. Retrieved from http://thejournal.com/Articles/2009/01/01/A-Second-Life-ForEducators.aspx?Page $=1$.

13. Wong, G. (2006). Educators explore "Second Life" online. In CNN. Retrieved from http://www.cnn.com/2006/TECH/11/13/second.life.university/. 